Acknowledgements: MD. PhD Constantin V. Kushnir, Main hospital of police.

Disclosure of Interests: None declared

DOI: 10.1136/annrheumdis-2021-eular.2931

\section{POS1400 A SYSTEMATIC REVIEW OF INTERNATIONAL GUIDELINES REGARDING THE ROLE OF RADIOGRAPHY IN THE DIAGNOSIS OF OSTEOARTHRITIS}

C. Henry-Blake ${ }^{1}$, K. Treadwell ${ }^{1}$, S. Parmar ${ }^{1}$, J. Higgs ${ }^{1}$, M. Marshall ${ }^{1}$, J. Edwards', G. Peat ${ }^{1}{ }^{1}{ }^{1}$ Keele University, Department of Primary, Community and Social Care, Keele, United Kingdom

Background: A substantial proportion of primary care osteoarthritis (OA) consultations are associated with an X-ray request ${ }^{(1,2)}$. Uncertainty exists regarding the ability of radiography to improve a clinical OA diagnosis, and the over-use of radiography may lead to inappropriate referrals due to severe radiographic features that do not correlate with patients' symptoms. Additionally, there are cost implications of unnecessarily imaging such a prevalent disease. As evidence questions the utility of routine radiography in $\mathrm{OA}$, the extent to which radiography is supported by international guidelines is unknown.

Objectives: To undertake a systematic review and narrative synthesis of UK and international guideline recommendations on the role of radiography in the diagnosis of $\mathrm{OA}$

Methods: A systematic search of eleven electronic databases (including EMBASE, MEDLINE CINAHL, Epistemonikos and Guideline Central) and the websites of nine professional organisations (including NICE, Royal College of Radiologists (RCR), EULAR, and the American College of Radiology $(A C R)$ ) identified the most recent evidence-based guidelines produced by professional organisations on the role of imaging in OA. Guidelines not addressing the role of radiography in the diagnosis of OA were excluded, as were non-English and spinal OA guidelines. Each title was screened by one reviewer whilst each abstract and full text underwent dual screening. A single reviewer, using a standard proforma, undertook data extraction. Each guideline was independently appraised by two reviewers using the AGREE II tool. A narrative synthesis of the nature and consistency of OA radiographic recommendations was performed.

Results: 18 evidence-based OA guidelines published between 1998-2019 were included. These guidelines considered OA at any joint $(n=8)$, or at the knee $(n=3)$, hip $(n=2)$, hand $(n=2)$, wrist $(n=1)$, foot $(n=1)$, and ankle $(n=1)$. Seven guidelines were produced by European organisations; four guidelines were produced by EULAR. Guidelines were targeted at general practitioners $(n=11)$, radiologists $(n=7)$, rheumatologist $(n=4)$ and orthopaedic surgeons $(n=3)$. Using the AGREE II tool, the identified guidelines scored highly on rigour of development (mean score 69\%) but poorly on applicability (32\%). All 18 guidelines recommended X-rays as the first-line modality, where imaging was indicated. A clinical diagnosis of OA without radiographic confirmation was recommended by all eleven guidelines produced by organisations represented general practitioners, with seven guidelines justifying this due to a poor correlation between radiographic features and clinical symptoms. Only three guidelines explicitly discouraged the routine use of radiography for the diagnosis of $O A$ and only two guidelines reassured practitioners of a low probability of missing serious pathology when not routinely requesting radiographs. Guidelines produced by organisations representing radiologists were more supportive of radiography. The ACR recommended radiographic confirmation in patients suspected to have OA at the hand, wrist, hip, knee, ankle, and foot. Conversely, the RCR recommended radiographic confirmation in patients suspected to have $\mathrm{OA}$ at the hand, feet, and hip, but not the knee.

Conclusion: Differences in guideline recommendations on the utility of radiography in OA appear related to country/region, professional organisation, and joint. The use and utility of radiography in OA may need to be reviewed in light of a shift towards remote consultations, a change that has been accelerated by COVID-19 in many countries.

REFERENCES:

[1] Yu D, Jordan K, Bedson J, Englund M, Blyth F, Turkiewicz A et al. Population trends in the incidence and initial management of osteoarthritis: age-period-cohort analysis of the Clinical Practice Research Datalink, 1992-2013. Rheumatology. 2017;56(11):1902-1917.

[2] Brand C, Harrison C, Tropea J, Hinman R, Britt H, Bennell K. Management of Osteoarthritis in General Practice in Australia. Arthritis Care \& Research. 2014;66(4):551-558

Acknowledgements: JJE is funded by an Academic Clinical Lectureship from the National Institute for Health Research (NIHR) for this research project (CL2016-10-003). The views expressed in this publication are those of the author(s) and not necessarily those of the NIHR, NHS or the UK Department of Health and Social Care.

Disclosure of Interests: None declared

DOI: 10.1136/annrheumdis-2021-eular.3117

\section{POS1401 ASSESSMENT OF INTERREADER RELIABILITY IN SCORING PATIENTS WITH HAND OSTEOARTHRITIS AND PSORIATIC ARTHRITIS BY FLUORESCENCE OPTICAL IMAGING}

B. Drude ${ }^{1}, \varnothing$. Maugesten ${ }^{2}$, S. G. Werner ${ }^{3}$, G. R. Burmester ${ }^{1}$, J. Berger $^{4}$, I. K. Haugen ${ }^{2}$, S. Ohrndorf ${ }^{1}{ }^{1}$ Charité Universitätsmedizin Berlin, Department of Rheumatology and Clinical Immunology, Berlin, Germany; ${ }^{2}$ Diakonhjemmet Hospital, Department of Rheumatology, Oslo, Norway; ${ }^{3} \mathrm{RHIO}$ (Rheumatology, Immunology, Osteology) Duesseldorf, RHIO Research Institute, Duesseldorf, Germany; ${ }^{4}$ Xiralite GmbH, Head Development and Produktion, Berlin, Germany

Background: Fluorescence Optical Imaging (FOI) utilises the fluorophore indocyanine green (ICG) to reflect enhanced microcirculation in hand and finger joints due to inflammation.

Objectives: We wanted to assess the interreader reliability of $\mathrm{FOI}$ enhancement in patients with hand osteoarthritis (OA) and psoriatic arthritis (PsA). Furthermore, predefined typical morphologic patterns were included to determine the ability of FOI to discriminate between both diagnoses.

Methods: An atlas with example images of grade 0-3 in different joint groups and typical morphologic patterns ('streaky signals'[1], 'green/blue nail sign'[2], 'Werner sign'[3,4], and 'Bishop's crozier sign') of PsA and hand OA was created. Two readers scored all joints in both hands ( 30 in total) of 20 cases with hand OA and PsA. The cases were randomly mixed and both readers were blinded to diagnosis. Each joint was rated on a semiquantitative scale from 0 to 3 in five different images (PrimaVista Mode (PVM), phase 1, 2 (first and middle image), and 3) during the FOI sequence according to the scoring method FOIAS (fluorescence optical imaging activity score) $[1,3]$. Interreader reliability on scoring joint enhancement was calculated using linear weighted Cohen's kappa (к). Agreement on diagnosis (hand OA vs. PsA) and different morphologic patterns was assessed by calculating (regular) Cohen's kappa.

Results: Overall agreement on scoring joint enhancement (all phases) was substantial $(\kappa=0.75)$, with greatest consensus in phase 2 first $(\kappa=0.75)$ and lowest agreement in phase $1(\kappa=0.46)$. Reliability varied in different joint groups (wrist, MCP, (P)IP, DIP), with almost perfect overall agreement on PIP joint affection $(\kappa=$ $0.81)$, substantial agreement on wrist $(\kappa=0.69)$ and DIP joint affection $(\kappa=0.63)$ and moderate agreement on MCP joint affection $(\kappa=0.49)$ across all phases. Consensus on morphologic patterns showed overall fair agreement $(\kappa=0.37)$ with a similar kappa value on the ability to discriminate between both diagnoses $(\kappa=0.3)$. Conclusion: Joint enhancement in FOI can be reliably assessed using a predefined scoring method. The ability of FOI to differentiate between hand OA and PsA seems to be limited. Clearer definition and more training might be needed to better agree on morphologic patterns in FOI.

REFERENCES:

[1] Glimm AM, Werner SG, Burmester GR, et al. Ann Rheum Dis. 2016 Mar;75(3):566-570

[2] Wiemann O, Werner SG, Langer HE, et al. J Dtsch Dermatol Ges. 2019 Feb;17(2):138-148

[3] Werner SG, Langer HE, Ohrndorf S, et al. Ann Rheum Dis. 2012 Apr;71(4):504-510

[4] Zeidler H 2019. Fluoreszenzoptische Bildgebung. In: Zeidler H, Michel BA Differenzialdiagnose rheumatischer Erkrankungen 5. Aufl. Springer, Heidelberg, S. 88-89

Disclosure of Interests: Benedict Drude: None declared, Øystein Maugesten: None declared, Stephanie Gabriele Werner: None declared, Gerd Rüdiger Burmester: None declared, Jörn Berger Employee of: Xiralite GmbH, Ida K. Haugen: None declared, Sarah Ohrndorf: None declared DOI: 10.1136/annrheumdis-2021-eular.3145

\section{POS1402 HIGHER PREVALENCE OF SUBCLINICAL ATHEROSCLEROSIS IN PSORIATIC ARTHRITIS PATIENTS}

S. Lugo-Perez ${ }^{1}$, I. J. Colunga-Pedraza², D. Á. Galarza-Delgado ${ }^{2}$, J. R. AzpiriLópez $^{1}$, A. B. Rodriguez-Romero ${ }^{2}$, N. Guajardo-Jauregui ${ }^{2}$, D. E. Flores Alvarado $^{2}$, A. Cárdenas ${ }^{2}$, A. C. Garza Acosta ${ }^{3}$, O. Ilizaliturri Guerra², G. GarcíaArellano ${ }^{2}$. ${ }^{1}$ Hospital Universitario "Dr. José Eleuterio González", Universidad Autónoma de Nuevo León, Cardiology, Monterrey, Mexico; ${ }^{2}$ Hospital Universitario “Dr. José Eleuterio González", Universidad Autónoma de Nuevo León, Rheumatology, Monterrey, Mexico; ${ }^{3}$ Hospital Universitario "Dr. José Eleuterio González", Universidad Autónoma de Nuevo León, Radiology, Monterrey, Mexico

Background: Psoriatic arthritis (PsA) patients have a higher risk of developing a cardiovascular (CV) event than the general population due to an increased prevalence of traditional $\mathrm{CV}$ risk factors and to disease characteristics such as disease duration and activity. The carotid ultrasound (US) is a non-invasive diagnostic tool that can detect the presence of subclinical atherosclerosis which is directly associated with the risk of developing a CV event.

Objectives: The aim of this study is to compare the prevalence of subclinical atherosclerosis detected by carotid US in PsA patients and controls. 
Methods: This is a cross-sectional, observational, and comparative study. A total of seventy-five PsA patients aged 40-75 years old, who fulfilled the 2006 CASPAR criteria and seventy-five matched controls by age ( \pm 5 years), gender and comorbidities were recruited for this study. Patients with history of a previous CV event and pregnant women were excluded from this study. A high-resolution $B$ mode carotid US was performed in all study subjects by a certified radiologist. Subclinical atherosclerosis was defined as the presence of a carotid plaque (CP) or an increased carotid intima media thickness (cIMT). The presence of CP was defined as a cIMT $\geq 1.2 \mathrm{~mm}$ or a focal narrowing $\geq 0.5 \mathrm{~mm}$ in the surrounding lumen. An increased cIMT was considered as a value $\geq 0.8 \mathrm{~mm}$. Distribution was evaluated with the Kolmogorov-Smirnov test. Comparisons were done with $\chi 2$ test for qualitative variables and Student's t test and Mann-Whitney's $U$ test for quantitative variables. A $p$ value $<0.05$ was considered statistically significant. Results: There were no differences when comparing the demographic characteristics between both groups (Table 1). When comparing the carotid US findings, a statistically significant difference was found in the prevalence of $\mathrm{CP}$, which was higher in the PsA group (44.0\% vs $26.7 \%, p=0.026$ ), in the presence of unilateral CP $(25.3 \%$ vs $10.7 \%, p=0.019)$ and in the presence of subclinical atherosclerosis (52.0\% vs $34.7 \%, p=0.032$ ) (Figure 1 ).

Conclusion: The prevalence of subclinical atherosclerosis was higher in PsA patients than controls, and this could be attributed to an increase in the inflammatory burden of these patients. The carotid US should be considered as part of the CV evaluation in all PsA patients, identifying those who would benefit from an opportune treatment preventing the development of a CV event.

REFERENCES:

[1] Yim KM, Armstrong AW. Updates on cardiovascular comorbidities associated with psoriatic diseases: epidemiology and mechanisms. Rheumatol Int. 2017;37(1):97-105.

Table 1. Demographic and clinical characteristics of psoriatic arthritis patients and controls.

\begin{tabular}{|c|c|c|c|}
\hline & $\begin{array}{l}\text { PsA } \\
(n=75)\end{array}$ & $\begin{array}{l}\text { Controls } \\
(n=75)\end{array}$ & $p$ \\
\hline Age years, mean $\pm \mathrm{DE}$ & $53.89 \pm 10.59$ & $54.25 \pm 7.08$ & NS \\
\hline Female gender, $\mathrm{n}(\%)$ & $43(57.3)$ & $43(57.3)$ & NS \\
\hline T2DM, n (\%) & $16(21.3)$ & $15(20.0)$ & NS \\
\hline HTN, $n(\%)$ & $28(37.3)$ & $21(28.0)$ & NS \\
\hline Dyslipidemia, n (\%) & $33(44.0)$ & $28(37.3)$ & NS \\
\hline Obesity, n (\%) & $31(41.3)$ & $32(42.7)$ & NS \\
\hline Active smoking, $\mathrm{n}(\%)$ & $14(18.7)$ & $18(24.0)$ & NS \\
\hline $\mathrm{BMI} \mathrm{kg} / \mathrm{m}^{2}$, median (p25-p75) & $29.32(26.23-32.03)$ & $28.9(25.4-33.5)$ & NS \\
\hline Disease duration years, median (p25-p75) & $5.0(3.0-10.0)$ & - & - \\
\hline DAPSA, median (p25-p75) & $12.6(5.3-22.9)$ & - & - \\
\hline Glucocorticoids, $n(\%)$ & $10(13.3)$ & - & - \\
\hline MTX, n (\%) & $51(68.0)$ & - & - \\
\hline bDMARD, n (\%) & $28(37.3)$ & - & - \\
\hline
\end{tabular}

PsA, psoriatic arthritis; NS, not significant; T2DM, type 2 diabetes mellitus; HTN, hypertension; BMI, body mass index; DAPSA, disease activity for psoriatic arthritis; MTX, methotrexate; bDMARD, biological disease modifying antirheumatic drug

Figure 1. Carotid ultrasound findings in psoriatic arthritis patients and controls.

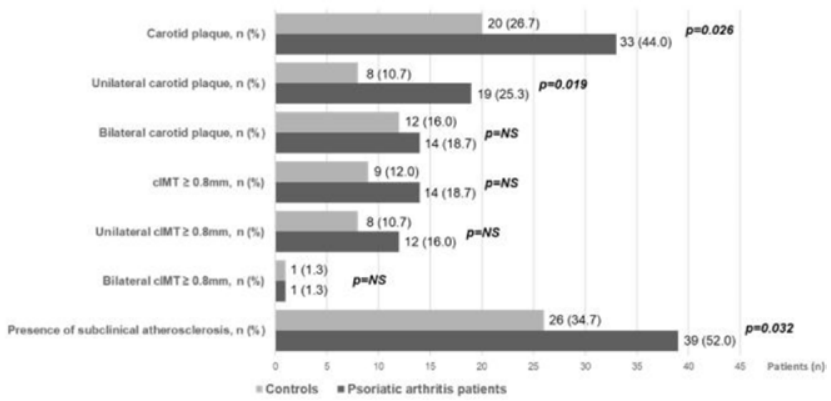

Disclosure of Interests: None declared

DOI: 10.1136/annrheumdis-2021-eular.3188

\section{POS1403 RADIOSYNOVECTOMY IN A CLINICAL SETTING: AN UNDEREXPLOITED TOOL AT OUR DISPOSAL}

L. R. Caballero Motta ${ }^{1}$, A. M. Anzola Alfaro ${ }^{1}$, J. Molina Collada ${ }^{1}$, I. Janta ${ }^{1}$, Y. K. Henao ${ }^{2}$, R. Perez Pascual ${ }^{2}$, F. J. López-Longo ${ }^{1}$, J. M. Alvaro Gracia ${ }^{1}$, J. C. Nieto González ${ }^{1}{ }^{1}$ Gregorio Marañón Hospital, Rheumatology, Madrid, Spain;

${ }^{2}$ Gregorio Marañón Hospital, Nuclear Medicin, Madrid, Spain
Background: Radiosynovectomy (RS) is a useful for treating inflammatory arthritis that fail conventional treatments. The main isotope used is Yttrium-90 on large joints as knees, whereas Erbium-169 and Renium-186 are more common in small and medium sized joints respectively.

$\mathrm{RS}$ is a safe procedure since the isotopes cannot escape the synovial capsule or be absorbed into circulation. It is, however, lethal against cells within the inflamed joint. The most common rheumatic disease treated with RS is rheumatoid arthritis (RA), followed by axial spondyloarthritis $(\mathrm{SpA})$ and idiopathic juvenile arthritis (JIA). It has also been used on persistent synovitis after joint replacements, pigmented villonodular synovitis (PVNS) and undifferentiated arthritis.

Objectives: To describe the experience in RS of a tertiary rheumatology center and compare patients with and without clinical response to treatment in the following 12 months. Methods: Observational retrospective study between May 31st 2013 and October 31st 2019. We collected demographic variables, data about the disease of the patient, the joints affected, isotope utilized, presence of Baker's cyst, systemic treatment received, need of additional infiltrations (before and after), complications and any changes in medication up to a year after the procedure.

All the RS were performed ambulatory and the radioisotope infiltration was guided by ultrasound, with $40 \mathrm{mg}$ of triamcinolone infiltrated after.

SPSS v23 was used for statistical analysis; with Chi2 for qualitative variables and Student's T distribution for quantitative variables.

Results: We evaluated 67 joints in 49 patients in total. All of them were refractory to conventional treatment. 44 patients $(65.7 \%)$ were women, median of 53.4 years of age (IQ 43.4-67.1)

The median disease duration was 12.5 years and RS seemed to fare better the longer the patient had the disease (median of 13.5 years vs 6.5 years $p<0.001$ ). The joints infiltrated where $46(68.6 \%)$ knees, $14(20.9 \%)$ wrists and $7(15.2 \%)$ elbows. Out of the knees, 16 (34.8\%) belonged to RA patients with effective response in $14(87,5 \%) .100 \%$ of elbows had an effective response, of them 6 $(85.7 \%)$ had RA. However, even when $9(64.2 \%)$ wrists also had RA as diagnosis, only $3(21.4 \%)$ were effective.

Of the PVNS, 6 out of 8 (75\%) had no clinical response, as shown in Table 1.

Table 1. RS response compared to clinical diagnosis.

\begin{tabular}{lllll}
\hline & TOTAL & EFFECTIVE INEFFECTIVE p \\
\hline & 67 & $46(68.6 \%)$ & $21(31.3 \%)$ & \\
& $(100 \%)$ & & & \\
Inflammatory Arthritides & $52(77.6)$ & $\mathbf{3 9}(\mathbf{7 5 \% )}$ & $13(25 \%)$ & $<0.0001$ \\
& & & & \\
(RA + PsA + SpA + SJIA), (\%) & & & & \\
RA (\%) & $30(44.7)$ & $\mathbf{2 2}(\mathbf{7 3 . 3})$ & $8(26.6)$ & $<0.001$ \\
RA positive ACPA/FR & $21(70)$ & $\mathbf{1 5}(\mathbf{7 1 . 4 )}$ & $6(28.6)$ & $<0.0001$ \\
Psoriasic arthritis (PsA) (\%) & $6(9)$ & $4(66.6)$ & $2(33.3)$ & 0.42 \\
SpA (\%) & $10(14.9)$ & $8(80)$ & $2(20)$ & 0.45 \\
sJIA (\%) & $6(9)$ & $5(83.3)$ & $1(16.6)$ & 0.55 \\
PVNS (\%) & $8(11.9)$ & $2(25)$ & $6(75)$ & $<0.001$ \\
Inespecific monoarthritis (\%) & $3(4.4)$ & $3(100)$ & $0(0)$ & 0.23 \\
OA + Calcium Pyrophosphate Deposition & $4(5.9)$ & $2(50)$ & $2(50)$ & 0.33 \\
(CPPD) (\%) & & & & \\
\hline
\end{tabular}

Intra articular corticosteroids were needed before RS, with no differences in effective and ineffective joints; however after RS it was significantly lower in effective joints in the first six months ( $0 \%$ vs $43 \% p<0.0001)$ and remained so in the following 6 months ( $0 \%$ vs $19 \% p<0.0001)$ Only $13(28 \%)$ patients with effective RS needed to change systemic treatment compared to 10 $(43 \%)$ of those ineffective $(p<0.0001)$. None of the patients with RS had any complication after the procedure during follow up.

Conclusion: Our study showed that knees were the main joint infiltrated and they had an overall good response to treatment, especially if the diagnosis was RA. Patients with effective procedures needed leest treatment changes and significantly less corticosteroids infiltrations.

In our study, RS in PVNS was significantly less effective than in inflammatory arthritis ( $25 \%$ vs $75 \% \mathrm{p}<0.0001$ ) and RA seemed to have the best response overall.

\section{REFERENCES:}

[1] Liepe K. Efficacy of radiosynovectomy in rheumatoid arthritis. Rheumatol Int. 2012 Oct; 32(10):3219-24.

[2] Ćwikła JB, Żbikowski P, Kwiatkowska B, Buscombe JR, Sudoł-Szopińska I. Radiosynovectomy in rheumatic diseases. J Ultrason. 2014 Sep; 14(58):241-51.

Disclosure of Interests: None declared

DOI: 10.1136/annrheumdis-2021-eular.3250

\section{POS1404 ECHOCARDIOGRAPHIC FINDINGS IN SYSTEMIC LUPUS ERYTHEMATOSUS PATIENTS AND ITS RELATION TO ANTIBODIES TITERS}

A. B. Rodriguez-Romero ${ }^{1}$, J. R. Azpiri-López ${ }^{2}$, I. J. Colunga-Pedraza ${ }^{1}$, D. Á. Galarza-Delgado ${ }^{1}$, S. Lugo-Perez ${ }^{2}$, N. Guajardo-Jauregui ${ }^{1}$, A. Cárdenas ${ }^{1}$, H. Azpiri-Diaz ${ }^{2}$, O. A. Cepeda-Ayala ${ }^{2} .{ }^{1}$ Hospital Universitario "Dr. José Eleuterio 